\title{
ACREDITACIÓN, EDUCACIÓN SUPERIOR Y RESPUESTA DEL PROFESORADO ENTRE 2008-2017
}

\section{ACCREDITATION, HIGHER EDUCATION AND TEACHER RE- SPONSE BETWEEN 2008-2017}

\author{
Querubín Patricio Flores Núñez ${ }^{1}$ \\ Carme Armengol Asparó ${ }^{2}$ \\ Joaquín Gairín Sallán ${ }^{3}$
}

Recibido, 20 de marzo de 2019

Aprobado, 20 de mayo de 2019

DOI: $10.25087 /$ resur7a2

\section{Resumen}

La investigación realizada en la República del Ecuador, se enfoca en analizar los procesos de acreditación de la educación superior y la respuesta de los profesores de la Pontificia Universidad Católica (PUCE) y sus sedes (Esmeraldas, Manabí, Santo Domingo de los Tsáchilas, Ambato, Ibarra y Quito) durante el período 2008-2017. El método es cuantitativo, alcance correlacional, diseño transversal longitudinal mediante la aplicación de una encuesta sobre tiempo de dedicación y producción científica a 513 docentes. Los datos evidencian que la acreditación de la educación superior del país inciden positivamente en la respuesta de los profesores en la producción académica.

Palabras clave: Acreditación, producción académica, educación superior, modelo, aseguramiento.

\begin{abstract}
The research conducted in the Republic of Ecuador, focuses on analyzing the processes of accreditation of higher education and the response of professors of the Pontifical Catholic University (PUCE) and its headquarters (Esmeraldas, Manabí, Santo Domingo de los Tsáchilas, Ambato , Ibarra and Quito) during the period 2008-2017. The method is quantitative, correlational scope, longitudinal cross-sectional design by applying a survey on time of dedication and scientific production to 513 teachers. The data show that the accreditation of higher education in the country has a positive impact on the response of teachers in academic production.
\end{abstract}

Palabras clave: Accreditation, academic production, higher education, model, assurance.

\footnotetext{
${ }^{1}$ Doctor en Educación e Investigador de la Pontificia Universidad Católica del Ecuador. Pedagogo, graduado en Ciencia Política y Profesor Universitario. - qpflores@puce.edu.ec

2 Profesora e investigadora de la Universidad Autónoma de Barcelona -UAB- Barcelona, España. - carme.armengol@uab.cat

${ }^{3}$ Doctor en Educación. Catedrático de la Universidad Autónoma de Barcelona. Profesor de Primaria, Graduado Social, Psicólogo y Pedagogo, actualmente Catedrático de Didáctica y Didáctica y Organización escolar - joaquin.gairin@uab.cat
} 


\section{INTRODUCCIÓN}

La Constitución del Estado ecuatoriano, adoptada mediante referéndum en septiembre del año 2008, plantea una transformación del sistema de educación superior. El Plan Nacional de Desarrollo, 2013-2017 acogiendo los postulados de la Conferencia Mundial sobre Educación superior sustenta el cambio en seis ejes fundamentales:

1. "igualdad de oportunidades,

2. calidad en la producción y transferencia del conocimiento,

3. pertinencia en el sentido de articular la educación con los grandes objetivos nacionales,

4. autodeterminación en la generación y aplicación del conocimiento,

5. articulación del sistema universitario con los diferentes niveles del sistema educativo,

6. autonomía bajo los principios de responsabilidad social y rendición de cuentas”(p.190).

Si nos centramos en el eje número 6 vemos que en Ecuador no se han realizado estudios a profundidad que comprueben la eficacia de la implementación de sistemas y modelos de aseguramiento de la calidad de las universidades (Lemaître, 2005; Avilés, 2016; Marginson, 2016). Esta carencia de conocimiento detallado sobre la universidad ecuatoriana urge la necesidad de estudios para determinar los resultados de la ingentes inversiones realizadas en el sistema de educación superior (Vélez \& Vélez León, 2008; Toscanini Segale, Aguilar Guzmán, \& García Sánchez, 2016; Avalos, Sánchez, \& Jara, 2016).

El objetivo de la investigación es explicar si el diseño institucional que reglamenta la educación superior en Ecuador fomenta la participación de los docentes de la Pontificia Universidad Católica del Ecuador en actividades de producción académica y científica. En este sentido se pretende responder a la pregunta ¿cómo el marco legal sobre educación superior promueve la respuesta del profesorado de la PUCE y el aumento o reducción de la producción académica en el período de 2008 a 2017?

Este estudio queda delimitado por el modelo de evaluación de la calidad de la educación superior, el plan nacional de desarrollo fundamentado en los principios del BUEN VIVIR , las políticas educativas, y el sistema de evaluación institucional.

\section{REFERENTES CONCEPTUALES}

\section{Motivaciones institucionales para acreditar la universidad en Ecuador}

Los estudios sobre acreditación nacionales e internacionales evidencian que es necesario poner atención a la vida universitaria para determinar la incidencia de la universidad en el contexto de su desarrollo. Sin embargo, es necesario definir con datos concretos las relaciones de causalidad existente entre las políticas institucionales y las consecuencias en la respuesta de las universidades en aspectos de docencia, investigación y producción científica (Barbón Pérez \& Fernández Pino, 2018).

El interés por el estudio de la acreditación en el sistema universitario ecuatoriano surge porque en los 10 años de gobierno de la Revolución Ciudadana se ha promovido cambios sociales, políticos y económicos. La movilización del cambio institucional universitario motiva el estudio para dar cuenta de lo que se realiza en la gestión académica y el uso adecuado de los recursos, autoevaluar las prácticas y repensar a la universidad (Cañón Pinto, 2005; Marginson, 2016).

En los últimos 5 años de activación del sistema de acreditación de la educación superior, las comisiones de evaluación han creado muchos recursos y sistemas de gestión para la acreditación tanto de programas como de Instituciones de Educación Superior (IES) y han fortalecido el desarrollo de una cultura de la calidad al interior de las IES, especialmente en los docentes que 
incoporaron el concepto de aseguramiento de la calidad en sus actividades académicas y científicas. Estos procesos de acreditación se ha convertido en acciones que aspiran crear una dinámica propia de la gestión de la información, la comunicación, los aprendizajes, los conocimientos, el empoderamiento, la representación y la participación (Rodríguez-Ponce, Pedraja-Rejas, AranedaGuirriman, González-Plitt, \& Rodríguez-Ponce, 2011).

Los procesos de aseguramiento de la calidad institucional en general y la acreditación de universidades en particular gozan de especial atención en la verificación de mecanismos de logro de los objetivos nacionales. Esto quiere decir que el establecimiento de un conjunto de mecanismos y políticas que regulen la calidad interna de la universidad (Lemaître, 2005) y su gestión acorde al contexto socio económico, en la que se activen los sistemas de gestión de la evaluación de las carreras e instituciones universitarias (Lemaître, 2005; Rodríguez-Ponce, Pedraja-Rejas, Araneda-Guirriman, González-Plitt, \& Rodríguez-Ponce, 2011).

De esta manera, se comprende con mayor claridad las políticas que una universidad debe reglamentar para realizar gestión de calidad. En este sentido es posible pensar que con diseños institucionales propios las universidades encuentren el camino del aseguramiento de la calidad de sus servicios y signifique un aporte para la excelencia (Bandeira \& Rubaii, 2016; Toscanini Segale, Aguilar Guzmán, \& García Sánchez, 2016).

La participación tiene también una función directa en la aplicación de mecanismos causales de aseguramiento de la calidad por lo que en todas las instituciones de educación superior hay mayor involucramiento de los distintos actores entre los que se destacan los estudiantes y docentes (Martínez-Moscoso \& Montesinos, 2012; Avilés, 2016; Bandeira \& Rubaii, 2016).

La Secretaría Nacional de Educación Superior, Ciencia y Tecnología (SENESCYT) se ha demorado en asumir e integrar un diseño institucional de la educación superior pertinente en razón de provocar los cambios que requieren tiempos más largos para la transformación social (Hidalgo, 2011; Avilés, 2016). La universidad ecuatoriana, abandonada antes del gobierno de la "Revolución Ciudadana”, incorpora el sistema de gestión de la calidad de la universidad a través de la planificación, implementación y evaluación para garantizar educación superior de calidad (Toscanini Segale, Aguilar Guzmán, \& García Sánchez, 2016; Barbón Pérez \& Fernández Pino, 2018).

Hasta el año 2010 la creación de una universidad dependía de la disposición de recursos económicos lo que redundaba en enseñanza mediocre e incorporación de docentes no calificados. Estos centros universitarios utilizaron instalaciones improvisadas en predios inadecuados y ofrecimientos fraudulentos de títulos universitarios, en el menor tiempo posible, pero que, a la larga, resultaría una titulación no reconocida. En el lenguaje popular fueron conocidas con el nombre de "universidades de garaje" (Ramírez, 2012; Secretaria Nacional de Educación Superior, Ciencia y Tecnología, 2012; Villavicencio, 2012).

\section{Acreditación de la educación superior en Ecuador}

El Consejo Nacional de Evaluación y Acreditación (CONEA, 2003) define que la universidad es la institución que forma profesionales para fortalecer el aparato productivo y aporten a la economía del conocimiento. La universidad es la institución que está llamada a orientar la vida de la sociedad en todos los ámbitos. Por eso su misión fundamental es la docencia, investigación y vinculación la comunidad.

La universidad debe ser guiada por profesionales que demuestren conocimientos de gestión para la acción eficiente de sus productos y servicios en clave de atención de calidad a los estudiantes. La misión esencial las instituciones de educación superior es mostrar niveles de excelencia en sus servicios a través del mejoramiento permanente de los profesionales que hacen la docencia, la investigación y la vinculación con la sociedad. En consecuencia, esta situación se verá reflejada en más altos niveles de planificación, ejecución y evaluación de los procesos de enseñanza-aprendizaje (Dias Sobrinho, 2008; González \& Espinoza, 2008; Secretaria Nacional de Educación Superior, Ciencia y Tecnología, 2012).

La búsqueda de la calidad universitaria tiene directa relación con la actividad investigativa y el desarrollo científico para ponerlo al servicio de la sociedad. Todo esto se complementa con 
un vínculo planificado y sostenido con la vida social y cultural (Ruiz, Mas, Tejada, \& Navío, 2008; De Vergara, Suárez, \& Miranda, 2014).

En este contexto, la acreditación de calidad académica está relacionado con la actividad esencial de la universidad que reside en la convicción de provocar comprensiones desde el análisis de la situación cultural para la integración de sus valores en la práctica social (Rodríguez-Ponce, Pedraja-Rejas, Araneda-Guirriman, González-Plitt, \& Rodríguez-Ponce, 2011; Semradova \& Hubackova, 2014).

En el marco de la gestión de la calidad, los docentes buscan el mejoramiento científico y adoptan actitudes y valores que dan testimonio del ejercicio vocacional de desaprender para enseñar. El ambiente universitario es la base para la investigación y actividad con la comunidad de la universidad, y debe sostenerse en estructuras semánticas de eticidad como la base para la actividad científica de las universidades (Lemaître, 2005; Rodríguez-Ponce, Pedraja-Rejas, AranedaGuirriman, González-Plitt, \& Rodríguez-Ponce, 2011; Fabela-Cárdenas \& García-Treviño, 2014).

Los indicadores de calidad son la base para la evaluación de la calidad de los programas universitarios. La coherencia de los procesos académicos se hace relevante a nivel institucional por los ambientes que se constituyen para hacer posible el hecho social. En definitiva, lo que se busca es el mejoramiento de la calidad puesto en evidencia en evaluaciones internas y externas (Castillo, 2003; Elton, 2006;Centurión, 2008;Mas \& Tejada, 2013; Ruiz, Mas, Tejada, \& Navío, 2008).

La estandarización de la evaluación de programas e instituciones de educación superior son el marco referencial para comparar y confrontar las prácticas institucionales que se traduce en acciones que serán mediadas por las cualidades que evidencian los actores (Lemaître, 2005; Fabela-Cárdenas \& García-Treviño, 2014; Rosal Durán, 2017).

Los indicadores y estándares son las acciones que se realizan en la universidad. Mientras que a nivel institucional se valora las orientaciones teleológicas institucionales, la gestión del plan estratégico, el uso adecuado de los recursos financieros, el cuidado del bienestar estudiantil, la formación permanente del capital cognitivo de los docentes y la búsqueda permanente de investigación para la innovación (Toscanini Segale, Aguilar Guzmán, \& García Sánchez, 2016; Avilés, 2016; Gómez, Tolozano, \& Delgado, 2017; Martínez-Moscoso \& Montesinos, 2012).

En relación con el objeto de estudio que nos ocupa es clave mencionar algunos aspectos que circunscribe esta investigación: la universidad dispone del profesores calificados que se han formado para ejercer la docencia en concordancia a su campo amplio y específico de formación (De Vergara, Suárez, \& Miranda, 2014; García Gallegos, Sarmiento Berrezueta, \& Rodríguez, 2016, Barbón Pérez \& Fernández Pino, 2018).

En virtud de lo dicho, se definen algunos estándares como: que las insituciones de educación superior dispongan de estrutruras, modelos que guíen la vinculación y la gestión de los docentes con normativas que fortalezcan la formación e integridad profesional con planes de carrera adecuados a la realidad institucional y estatal (Toscanini Segale et al., 2016; Gómez et al., 2017; Granja, 2018).

Asimismo, que los docentes dispongan de una carga de trabajo dosificada en concordancia a su nivel profesional y distribuidas en actividades de docencia, investigación y vinculación. Además, los docentes también deben disponer de oportunidades para la actualización continua en su campo profesional y en docencia para la universidad mediante intercambio y movilidad nacional e internacional (Mag, 2014; González, 2014; Duţă \& Foloştină, 2014; Zlatić, Bjekić, Marinković, \& Bojović, 2014; Ruiz et al., 2008).

Por otra parte, hay que destacar que los ayudantes de cátedra deben recibir capacitación para la gestión de la clase y el manejo de recursos tecnológicos y otras competencias relativas a la gestión de los aprendizajes (Semradova \& Hubackova, 2014; Corcoran \& O’Flaherty, 2017; Patton \& Parker, 2017).

Los principios que orientan los procesos de acreditación de la calidad de la educación 
superior se fundamentan en la oferta de oportunidades para el ingreso a la universidad, sin discriminación, en búsqueda permanente del perfeccionamiento como seres humanos integrales que vivan en armonía con el entorno natural y social.

\section{METODOLOGÍA}

La metodología tiene un enfoque cuantitativo, de alcance correlacional; el diseño es narrativo; la herramienta de recolección de datos es una encuesta de 32 preguntas aplicada a 513 docentes del sistema nacional de la Pontificia Universidad Católica del Ecuador; y la técnica de análisis de los datos es la de series temporales que se define como un conjunto de datos obtenidos en períodos de tiempo regulares o cronológicos (Valderrey Sanz, 2010). El supuesto básico de los estudios basados en series temporales es que patrones de comportamiento pasado se repiten en el futuro (Valderrey Sanz, 2010). Por lo tanto, lo que se pretende es identificar las influencias para hacer proyecciones sobre la variable de estudio (Valderrey Sanz, 2010).

En este estudio se integran métodos y datos, a través de los cuales se procede a interpretar aspectos propios de la investigación para obtener mayor conocimiento del fenómeno.

El análisis de datos se ha realizado considerando que los ítems corresponden a las preguntas relacionadas con:

Tabla 1 Ítems y preguntas

\begin{tabular}{|l|l|}
\hline Item & \multicolumn{1}{|c|}{ Preguntas } \\
\hline p11 & Número de horas ((1-4), (5-8), (9-12), (13-16) de docencia \\
\hline p12 & Número de horas((1-4), (5-8), (9-12), (13-16) de investigación \\
\hline p13 & Número de horas((1-4), (5-8), (9-12), (13-16) de vinculación \\
\hline p21 & Vínculo de docentes con proyectos de investigación nacionales \\
\hline p22 & Vínculo de docentes con proyectos de investigación internacionales \\
\hline p23 & Artículos científicos indexados \\
\hline p24 & Artículos no indexados \\
\hline p25 & Publicación de libros \\
\hline p26 & Publicación de capítulos en libros \\
\hline p27 & Ponencias nacionales \\
\hline p28 & Ponencias internacionales \\
\hline p29 & Patentes \\
\hline p30 & Aportes (mapas, obras de arte, obras musicales), \\
\hline p31 & Aportes (consultorías, libros de texto manuales especializados). \\
\hline
\end{tabular}

Fuente: Elaboración propia

\section{RESULTADOS}

En el estudio han participado 513 profesores de los que 237 son mujeres (46,56\%) y 272 son hombres $(53,44 \%)$. Cuatro profesores no responden a este ítem. Si nos centramos en el tipo de contrato 428 trabajan tiempo completo, 28 medio tiempo y 53 tiempo parcial. Por tanto, se desprende que la mayor parte de los docentes encuestados dedican su trabajo a tiempo completo 
y son los que mayoritariamente están aportando a la producción científica en la Pontificia Universidad Católica del Ecuador.

En lo relativo a la cuestión de la categoría de los docentes, los datos revelan que 334 son docentes auxiliares, 107 agregados y 64 principales. Por tanto, se tiene que la mayor parte de los docentes encuestados que dedican su trabajo a tiempo completo son docentes auxiliares y que, junto a los docentes agregados, son los que mayoritariamente aportan a la en los indicadores de acreditación en la Pontificia Universidad Católica del Ecuador.

\section{Dedicación, docencia y producción científica}

La dedicación de los docentes a la actividad académica permite comparar la realidad de la producción científica y la formación de competencias para la excelencia en este ámbito del quehacer universitario. La producción científica se valora a través de los datos que se han obtenido gracias a las investigaciones que han realizado los docentes (Castañeda \& Castañeda, 2007) y su productividad define a la calidad institucional.

El objetivo de las universidades es generar conocimiento relacionado con la práctica científica de los docentes y se valora a través de la publicación de los productos que son resultado de la vinculación de los docentes en proyectos investigativos orientados a la producción de artículos, libros, patentes y otros aportes de relevancia. La PUCE asigna un número de horas a la actividad de docencia, investigación y gestión de procesos administrativos y valora la situación de la producción científica de los profesores con asignación de (1-4), (5-8), (9-12), (13-16) horas en el período de estudio.

La producción científica depende del tipo de dedicación y del tiempo asignado para estas actividades. Por esta razón, se realizan las relaciones existentes entre las horas asignadas a cada actividad y el dato obtenido.

En este sentido, el profesorado de la PUCE con asignación de (1-4), horas a la docencia que se involucra en proyectos de investigación promovidos por la universidad, crece en 5 puntos en el período de estudio, mientras que en proyectos internacionales el crecimiento es de 6 puntos. Los docentes con asignación de (5-8) horas aumenta su producción en 2 puntos con una severa caída entre los años 2016 y 2017, mientras que en proyectos internacionales la tendencia de crecimiento es de 20 puntos. Los docentes con asignación de (9-12) horas aumenta su producción en 4 puntos en proyectos de investigación internos con una severa caída entre los años 2016 y 2017, mientras que en proyectos externos la tendencia de crecimiento es de 14 puntos.

Los docentes con asignación de (13-16) horas, mantienen su producción en 6 puntos en proyectos nacionales con una severa caída entre los años 2016 y 2017, y con inmovilidad en todo el período de estudio, mientras que en proyectos internacionales la tendencia de crecimiento es de 11 puntos.

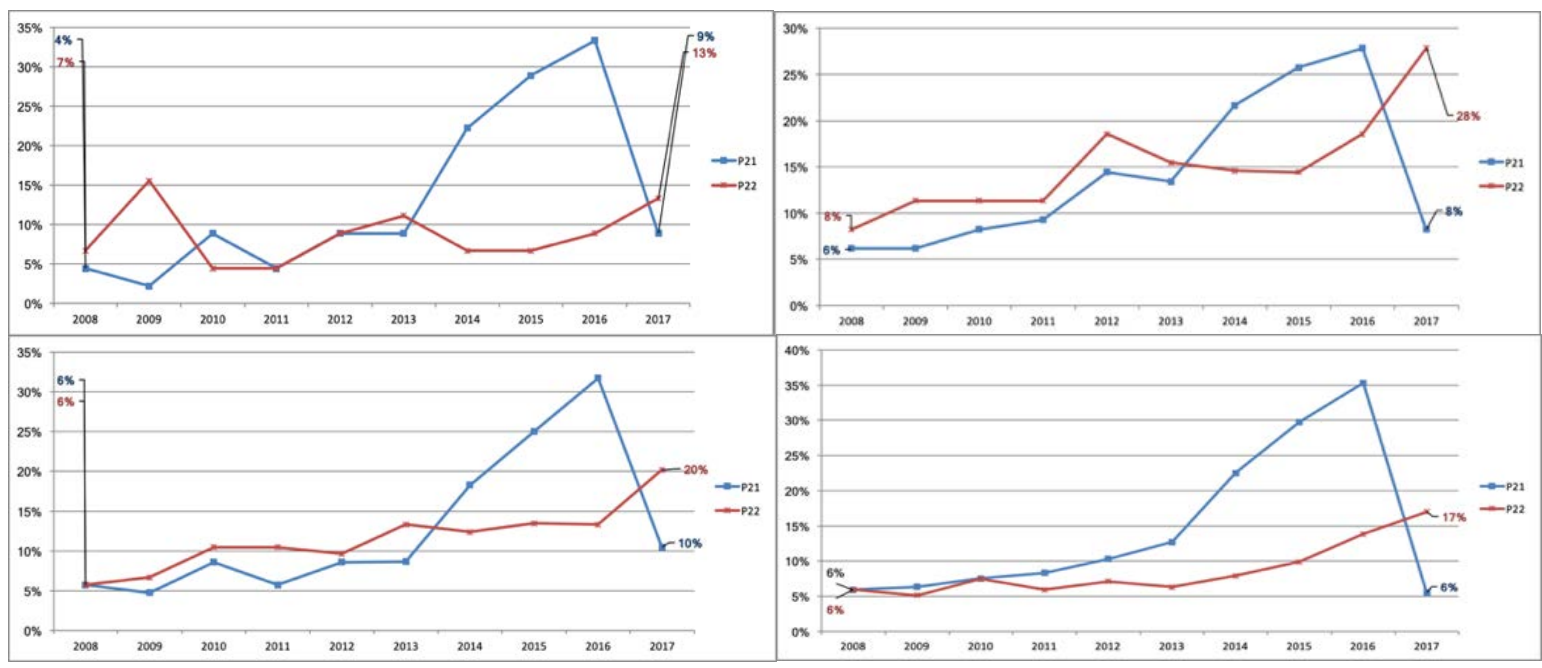

Gráfica 1: Horas dedicadas a la Docencia (1-4), (5-8), (9-12), (13-16) y P(21-P22). 
Fuente: elaboración propia (2018)

Por otra parte, la producción académica de los docentes con asignación de (1-4) horas en elaboración de artículos científicos indexados aumenta en 27 puntos, la publicación de artículos no indexados incrementa en 11 puntos, la publicación de libros en 7 puntos y capítulos en libros en 13 puntos.

La producción académica de los docentes con asignación de (5-8) horas en elaboración de artículos científicos indexados aumenta en 22 puntos, la publicación de artículos no indexados incrementa en 9 puntos, la publicación de libros en 4 puntos y capítulos en libros en 9 puntos.

La producción académica de los docentes con asignación de (9-12) horas en elaboración de artículos científicos indexados aumenta en 34 puntos, la publicación de artículos no indexados incrementa en 20 puntos, la publicación de libros en 6 puntos y capítulos en libros en 14 puntos.

La producción académica de los docentes con asignación de (13-16) horas en elaboración de artículos científicos indexados aumenta en 35 puntos, la publicación de artículos no indexados incrementa en 5 puntos, la publicación de libros en 1 puntos y capítulos en libros en 10 punto.

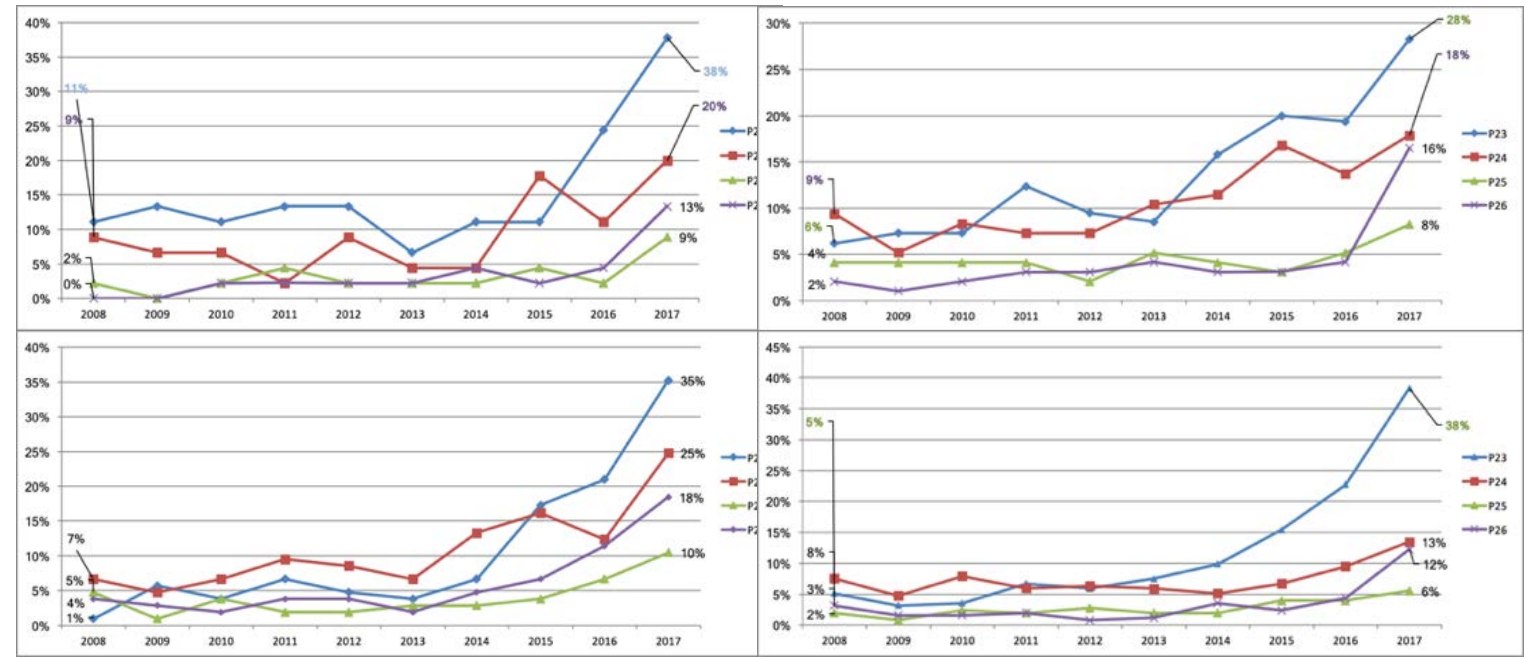

Gráfica 2: Horas dedicadas a la Docencia ((1-4), (5-8), (9-12), (13-16)) (P23-24-25-26)

Fuente: elaboración propia (2018)

En lo relativo a las ponencias nacionales, la producción del profesorado de la PUCE con asignación de (1-4), varía en 18 puntos y la de ponencias internacionales en 36 puntos.

En lo relativo a las ponencias nacionales, la producción de los docentes con asignación de (5-8) varía en 16 puntos y la de ponencias internacionales en 35 puntos. En lo relativo a las ponencias nacionales, la producción de los profesores con asignación de (9-12) varía en 25 puntos y la de ponencias internacionales en 27 puntos.

En lo relativo a las ponencias nacionales, la producción de los docentes con asignación de (13-16) varía en 16 puntos, y la de ponencias internacionales en 33 puntos en el período de estudio.

La participación de los docentes en actividad comunicacional es significativa en el período de análisis. 


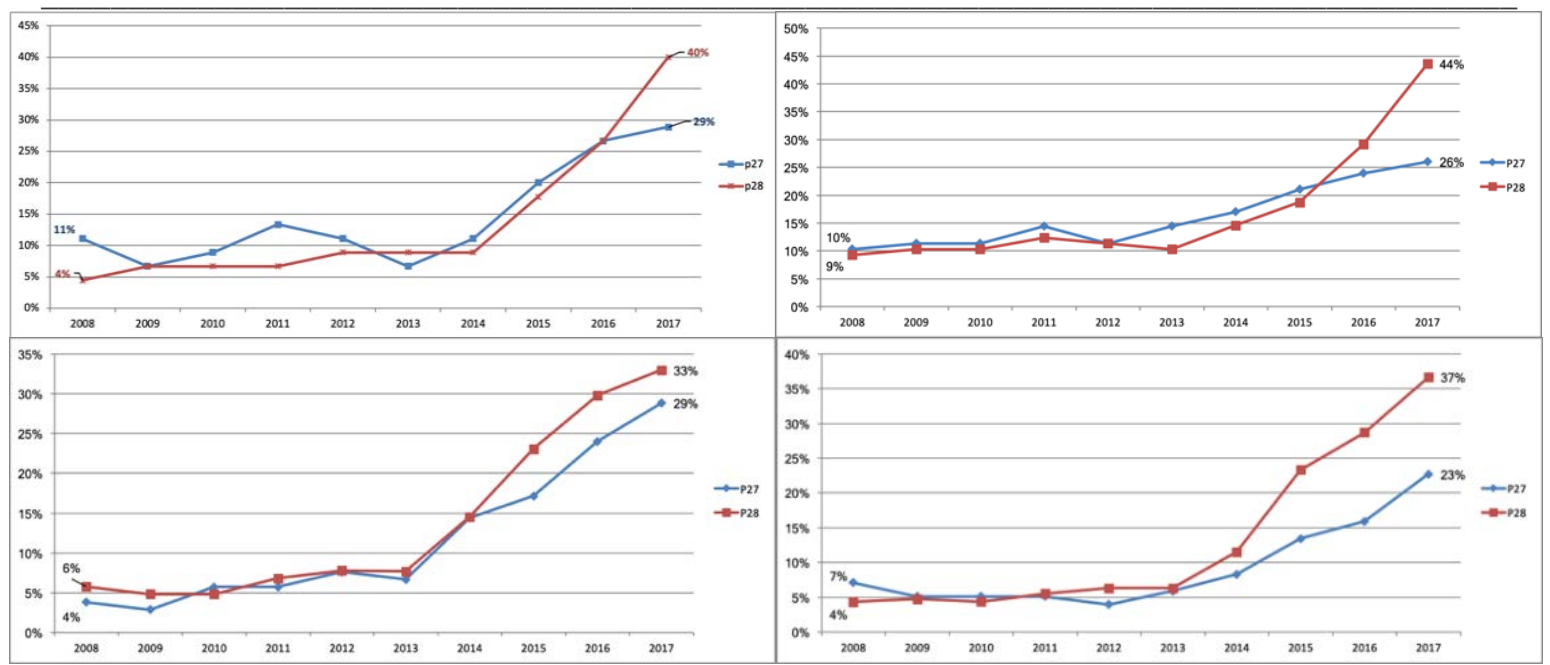

Gráfica 3: Horas dedicadas a la Docencia ((1-4), (5-8), (9-12), (13-16)) P(27-28)

Fuente: elaboración propia.

Finalmente, en lo relativo a los docentes con dedicación (1-4) horas, los datos evidencian un restringido crecimiento en el registro de patentes dado que entre 2015 y 2017 hay una subida de la producción de 5 puntos. La producción de aportes (mapas) varía en 9 puntos y otros aportes (consultorías) en 7 puntos.

Por otro lado, los datos de los docentes con dedicación (5-8) horas evidencian un restringido crecimiento en el registro de patentes, es decir, hay una subida, mínima, de la producción de 3 puntos. La producción de aportes (mapas) varía en 1 punto y otros aportes (consultorías) en 7 puntos.

En lo referente, a los docentes con dedicación (9-12) horas, los datos evidencian un restringido crecimiento en el registro de patentes, es decir, hay una subida, mínima, de la producción de 1 punto. La producción de aportes (mapas) varía en 2 puntos y otros aportes (consultorías) en 11 puntos.

Finalmente, los datos de los docentes con dedicación (13-16) horas evidencian un restringido crecimiento en el registro de patentes, es decir, hay una subida, mínima, de la producción de 2 puntos. La producción de aportes (mapas) varía en 4 puntos y otros aportes (consultorías) en 9 puntos.

Es necesario hacer notar por qué a partir del año 2016 se registra un despunte de los indicadores y se considera que la recategorización de la universidad podría ser un agente que incide en el comportamiento institucional.

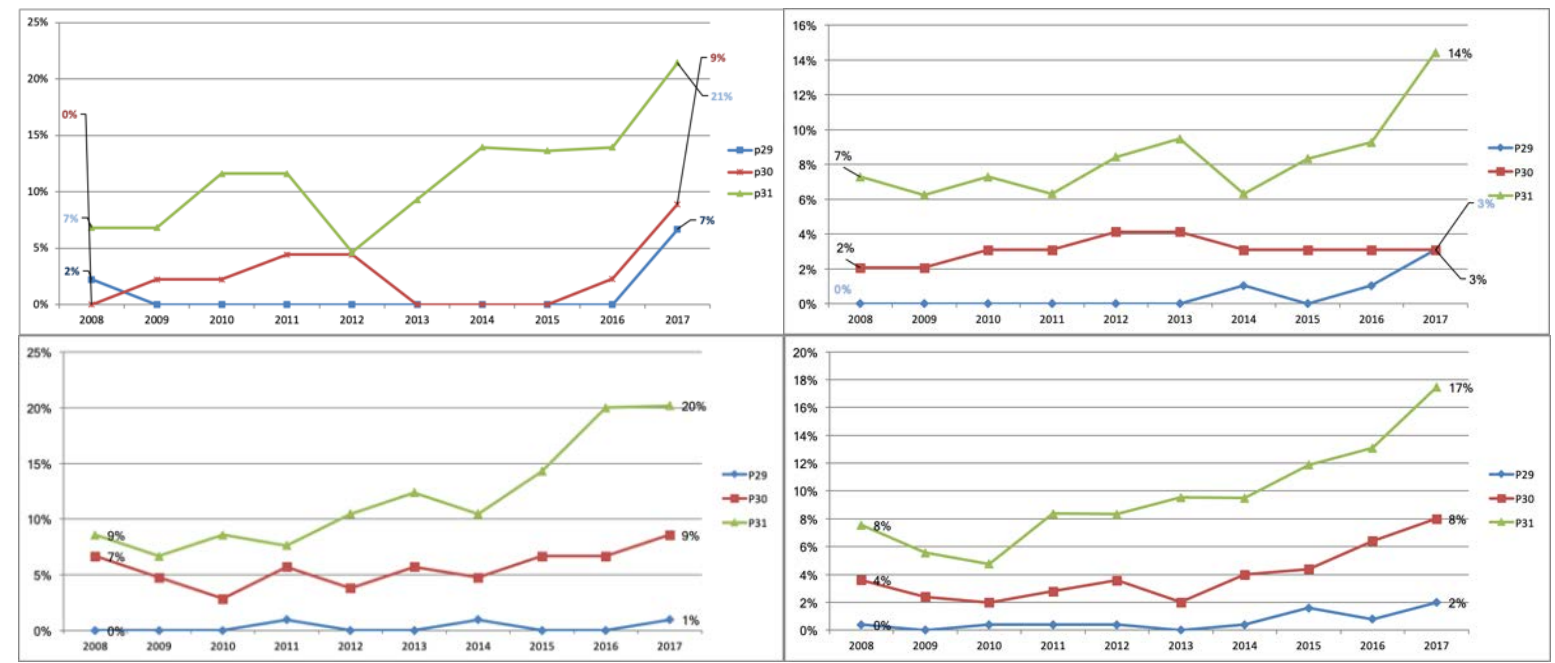

Gráfica 4: Horas dedicadas a la Docencia ((1-4), (5-8), (9-12), (13-16)) P(29-30-31)

Fuente: elaboración propia. 


\section{Tiempo de dedicación, investigación y producción científica}

En cuanto al profesorado de la PUCE con asignación de (1-4) horas a la investigación y que se vincula en proyectos de investigación promovidos por la universidad, crecen en 5 puntos en el período de estudio. En lo relativo al vínculo de los docentes que interactúan en proyectos investigativos externos el crecimiento es de 12 puntos en el período de análisis.

Los docentes con asignación de (5-8) horas a la investigación, vinculados en proyectos de investigación promovidos por la universidad, hay una reducción de la productividad en 2 puntos en el período de estudio. En el vínculo de los docentes que interactúan en proyectos investigativos externos el crecimiento es de 11 puntos en el período de análisis.

Los profesores con asignación de (9-12) horas a la investigación, vinculados en proyectos de investigación promovidos por la universidad, hay una reducción de la productividad en 4 puntos en el período de estudio. En el vínculo de los docentes que interactúan en proyectos investigativos externos el crecimiento es de 17 puntos.

El tiempo de dedicación de (9-12) horas a la investigación, vinculados en proyectos de investigación promovidos por la universidad, hay una reducción de la productividad en 3 puntos en el período de estudio. En el vínculo de los docentes que interactúan en proyectos investigativos externos el crecimiento es de 23 puntos.

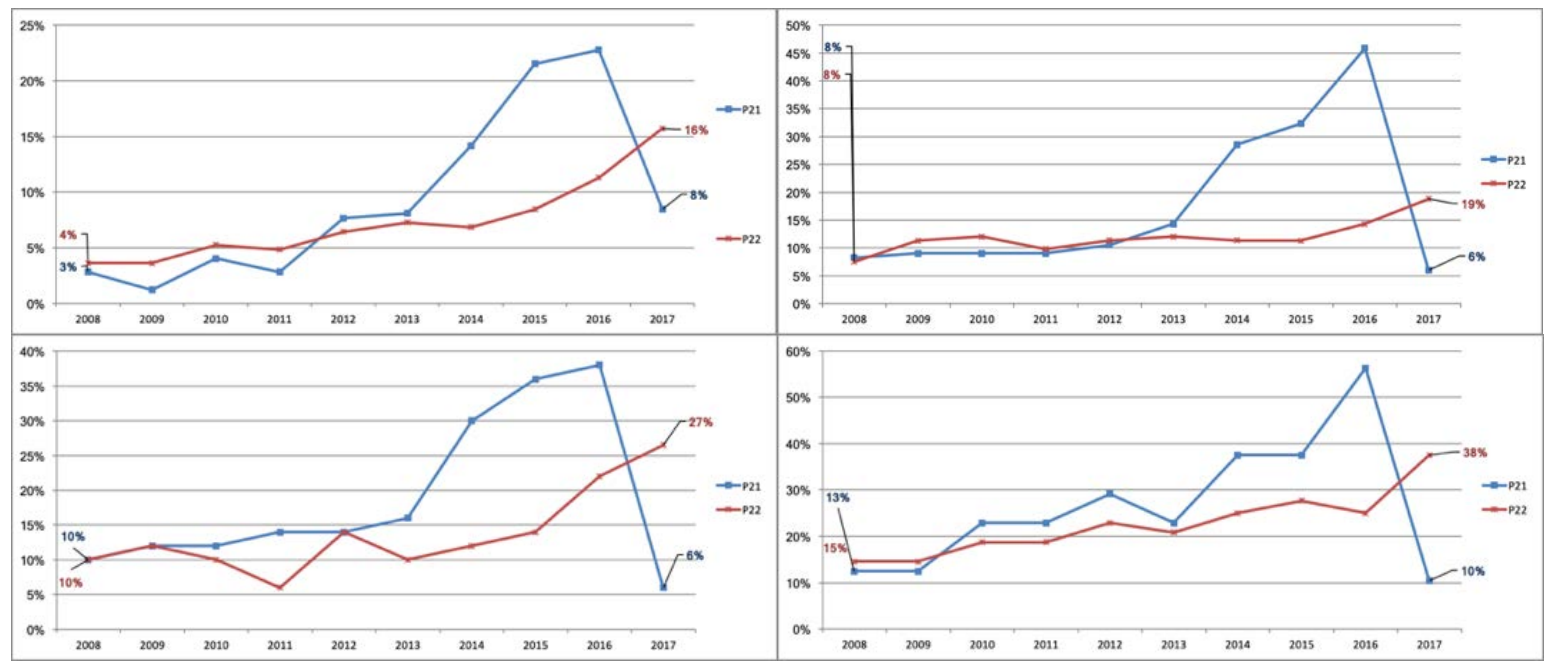

Gráfica 5: Horas dedicadas a la investigación ((1-4), (5-8), (9-12), (13-16)) P (21-2

La producción de artículos científicos de los docentes con asignación de (1-4) horas a la investigación aumentó en 6 puntos; de artículos no indexados aumentó en 9 puntos; la producción de libros aumentó en 25 puntos y los capítulos en libros en 13 puntos.

La producción de artículos científicos de los docentes con dedicación de (5-8) horas a la investigación aumentó en 38 puntos; de artículos no indexados aumentó en 19 puntos; la producción de libros aumentó en 4 puntos y los capítulos en libros en 12 puntos.

La producción de artículos científicos de los profesores con dedicación de (9-12) horas a la investigación aumentó en 49 puntos; de artículos no indexados aumentó en 10 puntos; la producción de libros aumentó en 2 puntos y los capítulos en libros en 4 puntos.

La producción de artículos científicos del profesorado con dedicación de (13-16) horas a la investigación aumentó en 38 puntos; de artículos no indexados aumentó en 23 puntos; la producción de libros aumentó en 4 puntos y los capítulos en libros en 17 puntos. 


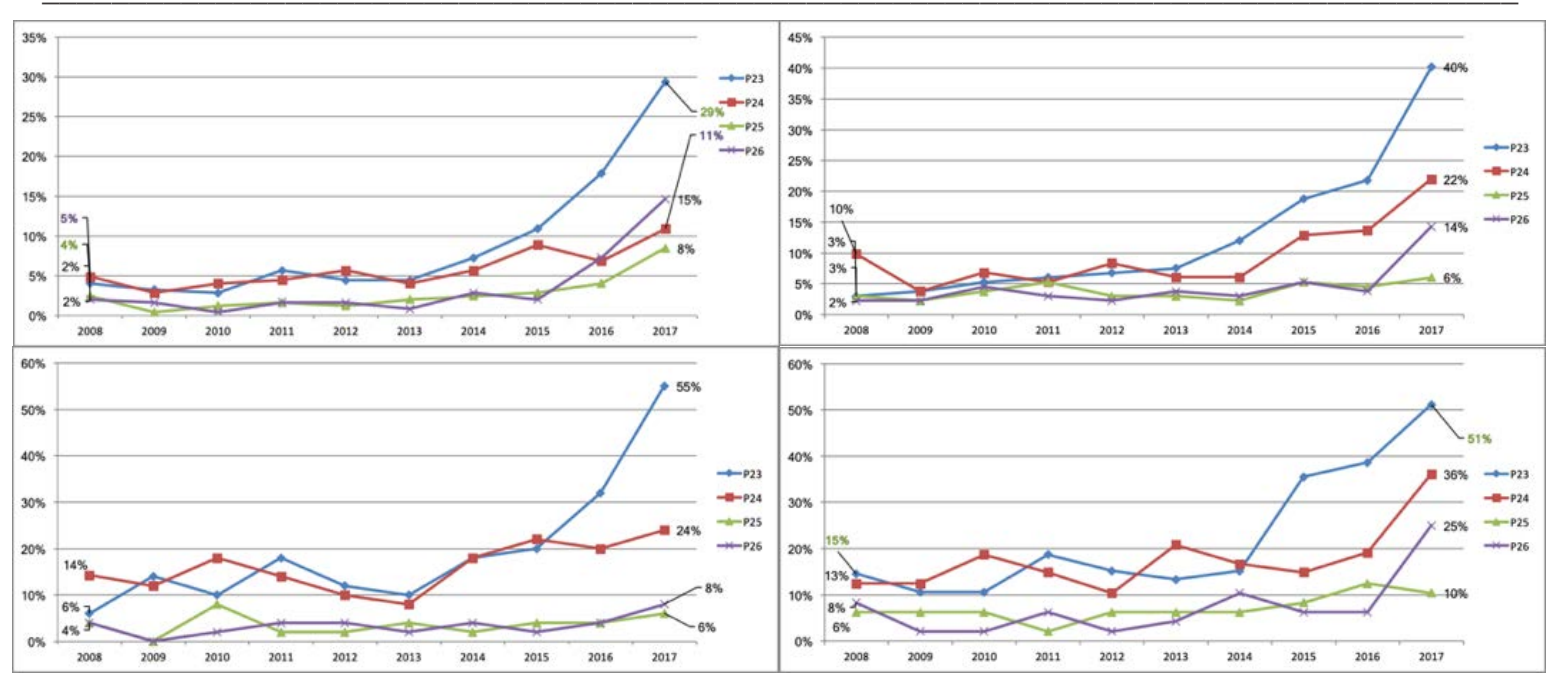

Gráfica 6: Horas dedicadas a la investigación ((1-4), (5-8), (9-12), (13-16)) P (23-24-25-25)

La presentación de ponencias internacionales de los docentes con asignación de (1-4) horas a la investigación aumentó en 15 puntos; ponencias nacionales aumentó en 24 puntos. La presentación de ponencias internacionales de los docentes con asignación de (5-8) horas a la investigación aumentó en 48 puntos; ponencias nacionales aumentó en 22 puntos. En la presentación de ponencias internacionales del profesorado con asignación de (9-12) horas a la investigación, aumentó en 26 puntos; ponencias nacionales aumentó en 29 puntos. La presentación de ponencias internacionales de los profesores con asignación de (13-16) horas a la investigación aumentó en 31 puntos; ponencias nacionales aumenta en 27 puntos.

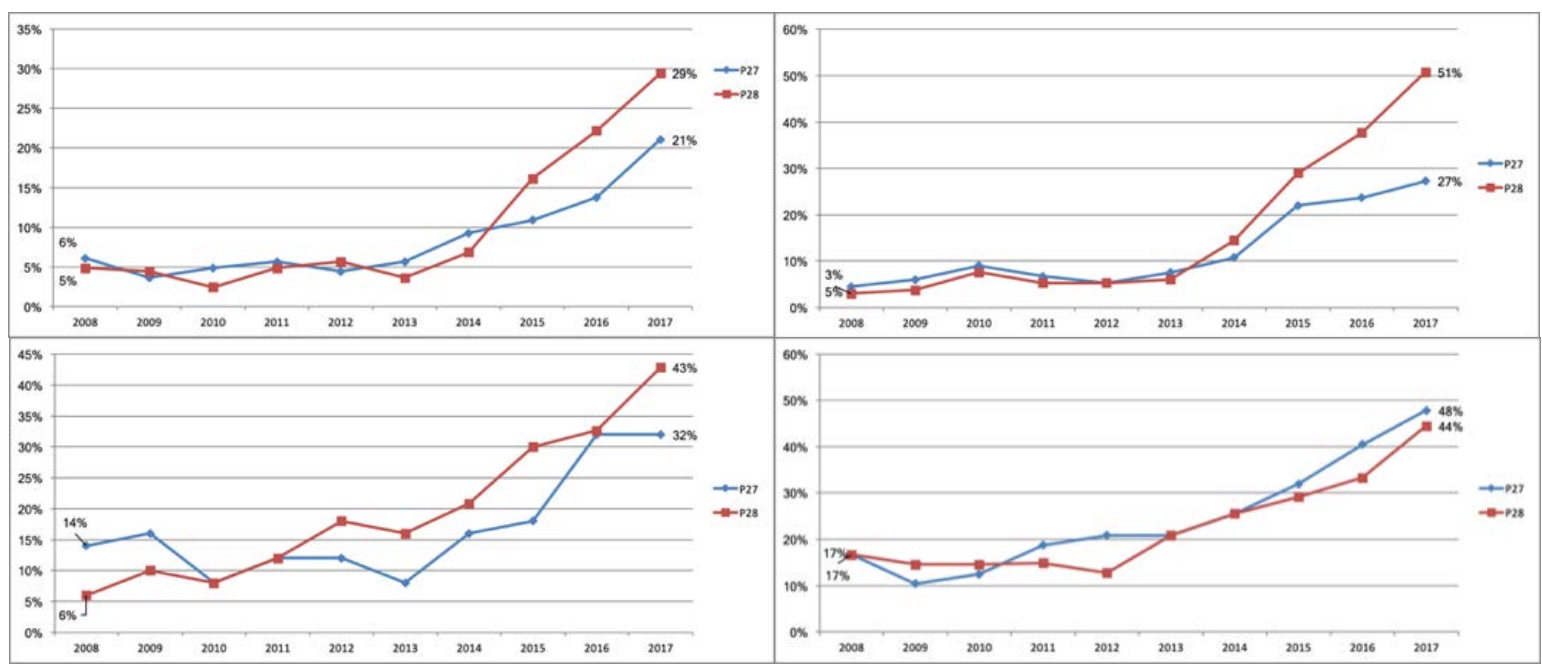

Gráfica 7: Horas dedicadas a la investigación ((1-4), (5-8), (9-12), (13-16)) P (27-28)

La producción de patentes de los docentes con asignación de (1-4) horas a la investigación aumentó en 4 puntos; otros aportes (mapas) aumentó en 4 puntos y otros aportes (consultorías) aumentó en 11 puntos.

La producción de patentes de los docentes con asignación de (5-8) horas a la investigación aumentó en 2 puntos; otros aportes (mapas) aumentó en 4 puntos y otros aportes (consultorías) aumentó en 10 puntos. La producción de patentes de los docentes con asignación de (9-12) horas a la investigación aumentó de 0 a 2 puntos; otros aportes (mapas) aumentó en 6 puntos y otros aportes (consultorías) aumentó en 9 puntos.

La producción de patentes de los docentes con asignación de (13-16) horas a la investigación se registró 0 crecimiento; otros aportes (mapas) aumentó en 11 puntos y otros aportes (consultorías) aumentó en 2 puntos. 


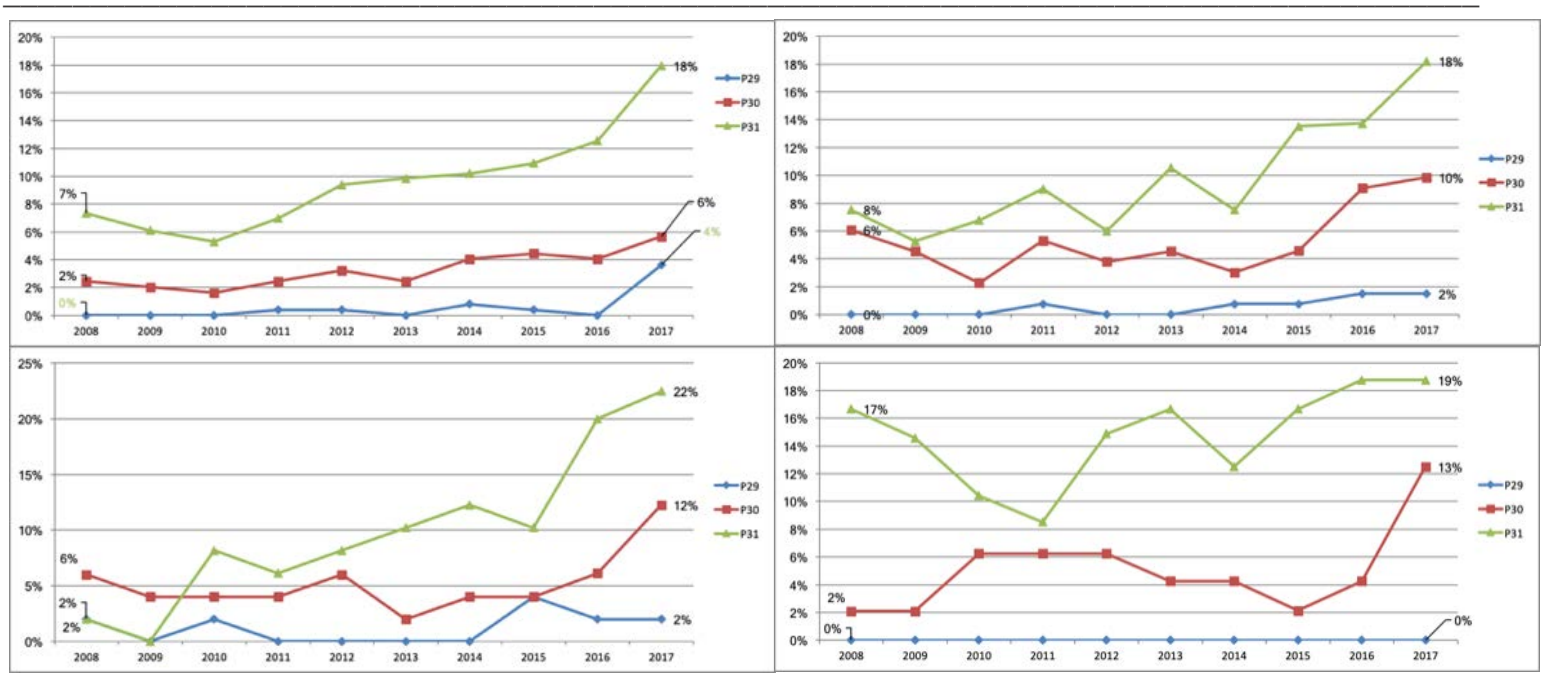

Gráfica 8: Horas dedicadas a la investigación ((1-4), (5-8), (9-12), (13-16)) P (29-30-31)

Tiempo de dedicación, gestión de procesos administrativos y producción científica

En cuanto al profesorado de la PUCE con asignación de (1-4) horas a la gestión de procesos administrativos vinculados en proyectos de investigación promovidos por la universidad, disminuye en 5 puntos y en lo relativo al vínculo de los docentes que interactúan en proyectos investigativos externos el crecimiento fue de 14 puntos en el período de análisis.

En cuanto a los docentes con asignación de (5-8) horas a la gestión de procesos administrativos vinculados en proyectos de investigación promovidos por la universidad, hubo una reducción de la productividad en 7 puntos en el período de estudio, en el vínculo de los docentes que interactúan en proyectos investigativos externos el crecimiento fue de 6 puntos en el período de análisis.

En cuanto a los profesores con asignación de (9-12) horas a la gestión de procesos administrativos vinculados en proyectos de investigación promovidos por la universidad, hubo una reducción de la productividad en 3 puntos en el período de estudio, en el vínculo de los docentes que interactúan en proyectos investigativos externos el crecimiento fue de 23 puntos.

En el tiempo de dedicación de (13-16) horas a la gestión de procesos administrativos vinculados en proyectos de investigación promovidos por la universidad, hubo un incremento de 7 y una reducción significativa entre el año 2016 y 2017 de 25 puntos en el período de estudio, en el vínculo de los docentes que interactúan en proyectos investigativos externos el crecimiento fue de 11 puntos.

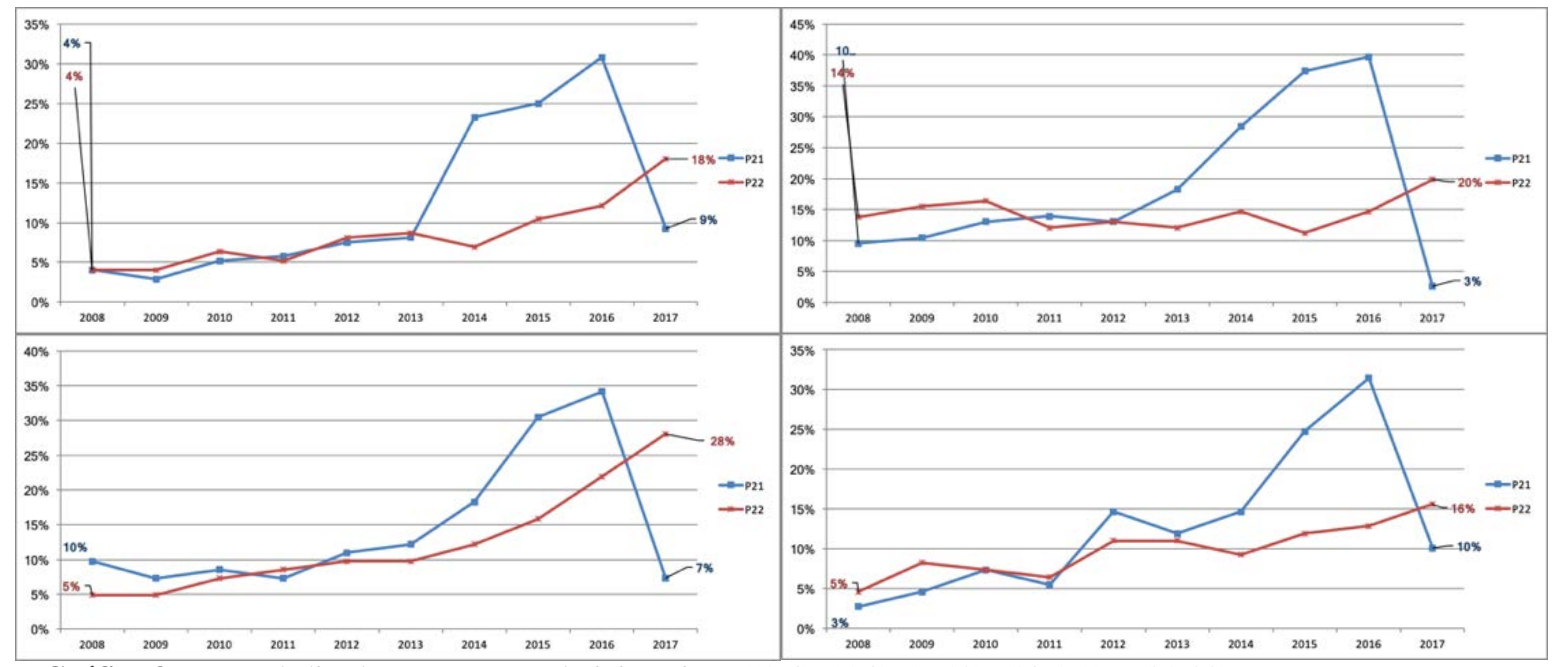

Gráfica 9: Horas dedicadas a procesos administrativos ((1-4), (5-8), (9-12), (13-16)) P (21-22)

La producción científica de artículos científicos de los docentes con asignación de (1-4) 
horas a la gestión de procesos administrativos aumentó en 21 puntos; de artículos no indexados aumentó en 9 puntos; la producción de libros aumentó en 7 puntos y los capítulos en libros en 11 puntos.

La producción de artículos científicos de los docentes con dedicación de (5-8) horas a la gestión de procesos administrativos aumentó en 35 puntos; de artículos no indexados aumentó en 14 puntos; la producción de libros no hubo crecimiento y los capítulos en libros en 7 puntos.

La producción de artículos científicos de los profesores con dedicación de (9-12) horas a la gestión de procesos administrativos aumentó en 41 puntos; de artículos no indexados aumentó en 16 puntos; la producción de libros aumentó en 6 puntos y los capítulos en libros en 20 puntos.

La producción de artículos científicos del profesorado con dedicación de (13-16) horas a la gestión de procesos administrativos aumentó en 38 puntos; de artículos no indexados aumentó en 11 puntos; la producción de libros aumentó en 7 puntos y los capítulos en libros en 12 puntos.

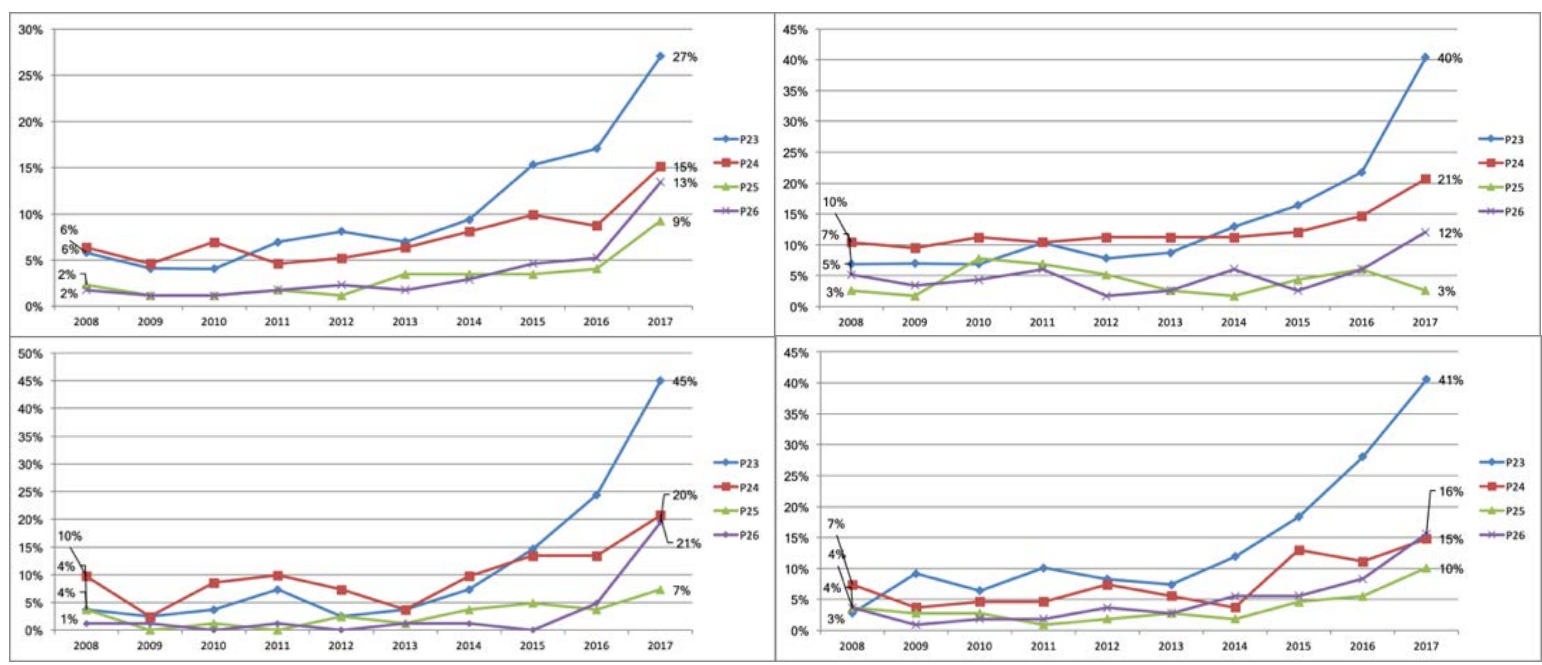

Gráfica 10: Horas dedicadas a procesos administrativos ((1-4), (5-8), (9-12), (13-16)) P (23-24-25-26)

La presentación de ponencias internacionales de los docentes con asignación de (1-4) horas a la gestión de procesos administrativos aumentó en 31 puntos; de ponencias nacionales aumentó en 23 puntos.

La presentación de ponencias internacionales de los docentes con asignación de (5-8) horas a la gestión de procesos administrativos aumentó en 13 puntos; de ponencias nacionales aumentó en 32 puntos.

La presentación de ponencias internacionales del profesorado con asignación de (9-12) horas a la gestión de procesos administrativos aumentó en 15 puntos; de ponencias nacionales aumentó en 34 puntos.

La presentación de ponencias internacionales de los profesores con asignación de (13-16) horas a la gestión de procesos administrativos aumentó en 23 puntos; de ponencias nacionales aumentó en 32 puntos. 


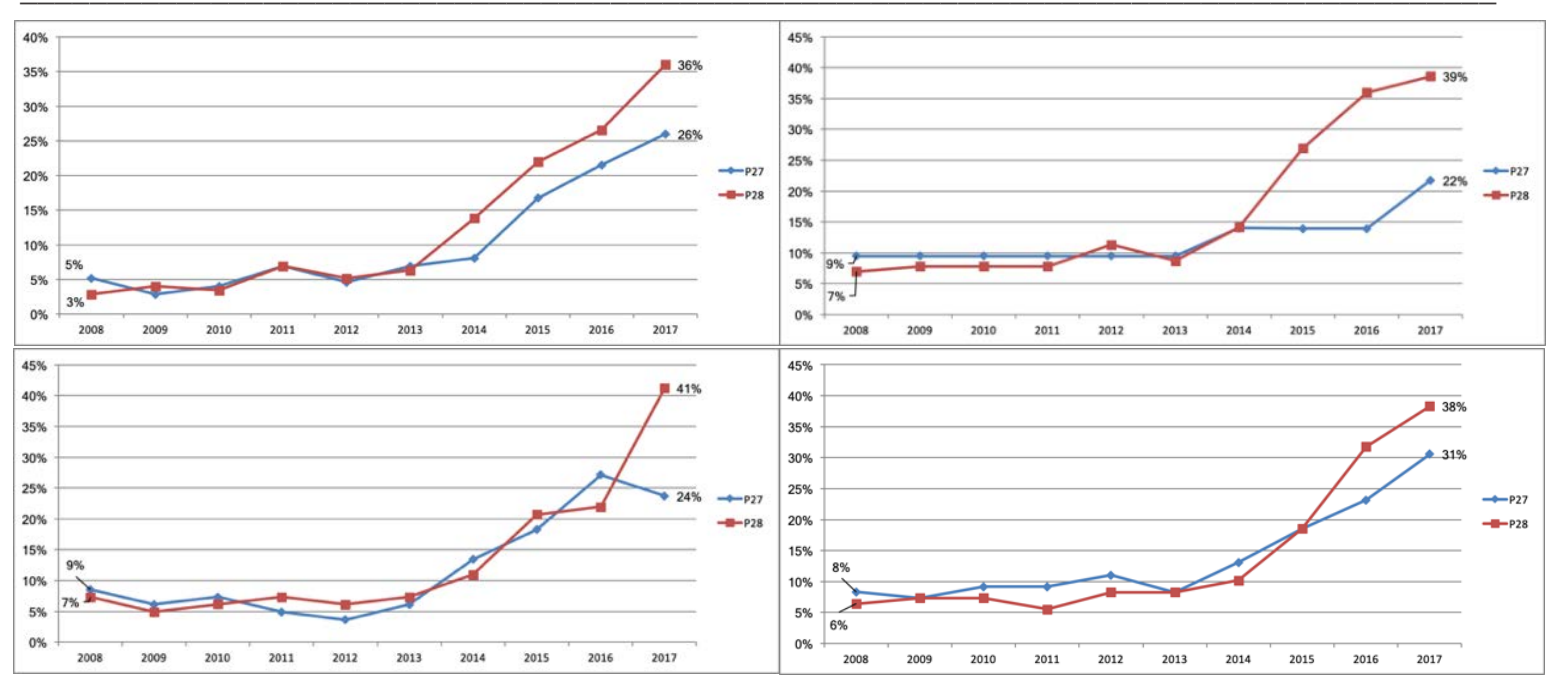

Gráfica 11 Horas dedicadas a procesos administrativos ((1-4), (5-8), (9-12), (13-16)) P (27-28)

La producción de patentes de los docentes con asignación de (1-4) horas a la gestión de procesos administrativos aumentó en 1 puntos; en otros aportes (mapas) amentó en 7 puntos y otros aportes (consultorías) aumentó en 16 puntos.

En la producción de patentes de los docentes con asignación de (5-8) horas a la gestión de procesos administrativos no se registró producción; otros aportes (mapas) aumentó en 5 puntos y otros aportes (consultorías) aumentó en 7 puntos.

La producción de patentes de los docentes con asignación de (9-12) horas a la gestión de procesos administrativos aumentó de 0 a 6 puntos; otros aportes (mapas) aumentó en 5 puntos y otros aportes (consultorías) aumentó en 9 puntos.

La producción de patentes de los docentes con asignación de (13-16) horas a la gestión de procesos administrativos incrementó en 4 puntos; en cuanto a otros aportes (mapas) no se registró incremento y se mantiene en 6 puntos y otros aportes (consultorías) aumentó en 4 puntos.

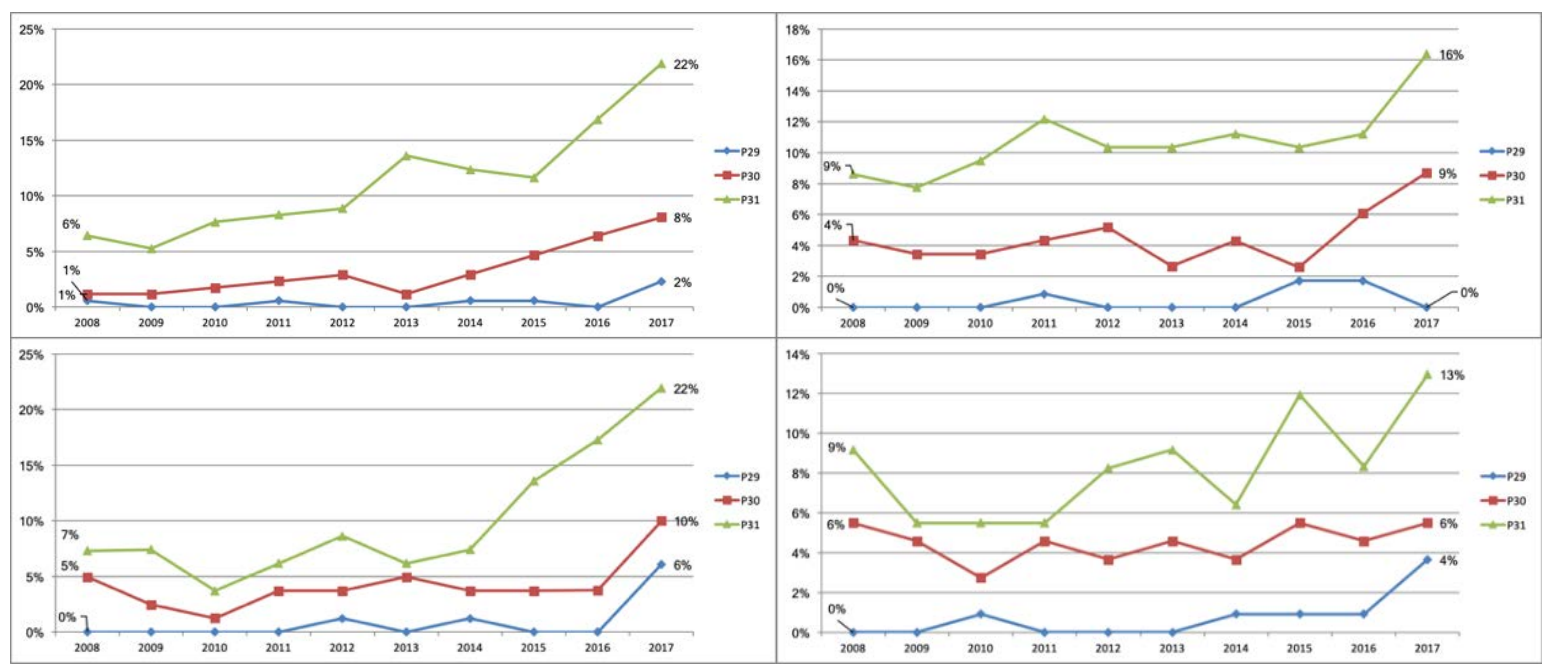

Gráfica 12: Horas dedicadas a procesos administrativos ((1-4), (5-8), (9-12), (13-16)) P (29-30-31)

\section{CONCLUSIONES}

Este estudio ha permitido conocer la respuesta de los docentes de la PUCE sobre la producción científica, mediante el registro del crecimiento y reducción de la actividad investigativa del profesorado en el período de 2008 a 2017. Los resultados evidencian que la normativa y la políticas institucionales han fomentado el crecimiento porcentual del desarrollo de la producción 
científica y se refleja en el número de las investigaciones y publicaciones. La vinculación de los docentes a proyectos nacionales e internacionales de investigación ha provocado el crecimiento de publicaciones como artículos científicos y libros de hasta el 35\%. Sin embargo, el crecimiento es mínimo en producción de patentes y aportes de relevancia y motiva a una adecuación de políticas internas y externas de gestión de la investigación y de la actividad científica, que involucre la publicación de resultados de las investigaciones con exigentes estándares de calidad.

Los resultados ponen de manifiesto que el tiempo de dedicación de los docentes a actividades de docencia, investigación y vinculación copan su desempeño profesional y el despliegue de actividades administrativas reducen su productividad. En este sentido, es indispensable considerar la reducción de la producción académica especialmente vinculada a la mayor cantidad de horas asignadas para actividades de investigación.

A nivel institucional, la PUCE, muestra algunos factores sobre los cuales es necesario trabajar con más meticulosidad: la diversidad del sistema universitario hace difícil la implementación de políticas unificadas en el diseño normativo que involucre a todos los profesores para que desarrollen actividades de docencia, investigación y vinculación con la sociedad de calidad. En definitiva, en la PUCE coexisten distintos modelos, concepciones, filosofías, creencias que dificultan el interés de unificar y homogeneizar una cultura organizacional que debe estar arraigada en los docentes y otros participantes clave como parte de la ruta para el nuevo modelo de universidad.

La investigación y la producción científica son actividades que se vinculan, por esto la publicación de libros, capítulos en libros, artículos científicos es resultado de la creatividad intelectual de los profesores y los procesos de investigación de la universidad. Una situación de la que depende la productividad científica en las universidades es la necesidad de procesos más eficientes de gestión de conocimiento para fortalecer a los investigadores para dar validez y confiabilidad a sus estudios y que los profesores publiquen en espacios de difusión de mayor impacto.

Un aspecto relevante del estudio es que el reglamento manda al profesor a realizar actividades de docencia, investigación y vinculación, sin embargo, la universidad no ha abordado el concepto de calidad de la dedicación de los docentes a su actividad académica. Los docentes realizan actividades que corresponden a intereses exclusivos de la unidad académica, proyecto personal o plan de la autoridad. 


\section{BIBLIOGRAFÍA}

Aguilar Gavira, S., \& Barroso Osuna, J. M. (2015). La triangulación de datos como estrategia en investigación educativa. https://doi.org/10.12795/pixelbit.2015.i47.05

Alcoreza, P. (2010). La revolución mundial del vivir bien. Plataforma Interamericana de Derechos Humanos, Democracia y Desarrollo. Retrieved from http://goo.gl/Tlxfc

Asamblea Nacional. (2008). Constitución Política del Ecuador 2008. Montecristi: Registro Oficial.

Avalos, R. B., Sanchez, J. C., \& Jara, A. V. (2016). Impacto de la evaluación y acreditación de las carreras profesionales ofertadas por la Universidad Nacional de Chimborazo desde la percepción de los estudiantes. Revista Ciencia UNEMI, 9(21), 36-47.

Avilés, G. T. (2016). Impacto de las Nuevas Políticas de Educación Superior en las Universidades y Escuelas Politécnicas del Ecuador. Revista Empresarial, 10(38), 28-34.

Bandeira, M. L., \& Rubaii, N. M. (2016). El valor del análisis de discurso en los estudios comparativos de políticas públicas. El caso de aseguramiento de calidad en la educación superior en Colombia y Ecuador. Estudios Políticos; Medellín, (49), 13-34. http://dx.doi.org.puce.idm.oclc.org/10.17533/udea.espo.n49a01

Barbón Pérez, O. G., \& Fernández Pino, J. W. (2018). Rol de la gestión educativa estratégica en la gestión del conocimiento, la ciencia, la tecnología y la innovación en la educación superior. Educación Médica, 19(1), 51-55. https://doi.org/10.1016/j.edumed.2016.12.001

Cañón Pinto, J. F. (2005). Internacionalización de la educación superior y educación superior internacional: elementos para un análisis sociológico general. Revista Colombiana de Sociología; Bogota, (25), 105-125.

Castañeda, G., \& Castañeda, R. (2007). Gerencia de investigación: Criterios gerenciales aplicados a la investigación. Ciencias Humanas, 2(6), 18-47.

Castillo, R. F. (2003). La calidad de la educación superior. Contextos: Revista de humanidades y ciencias sociales, (9), 97-104.

Centurión, D. (2008). La calidad de la educación superior. Revista Internacional de Investigación en Ciencias Sociales, 4(1), 73-96.

CONEA, C. N. de E. y A. de la E. S. del E. (2003, September). La calidad en la Universidad Ecuatoriana. Principios, características y estándares de Calidad.

Corcoran, R. P., \& O’Flaherty, J. (2017). Executive function during teacher preparation. Teaching and Teacher Education, 63, 168-175. https://doi.org/10.1016/j.tate.2016.12.023

De Vergara, D. L., Suárez, A. A. G., \& Miranda, A. J. M. (2014). Calidad de la Educación Superior: Un Análisis de sus principales determinantes. Saber, Ciencia y Libertas, 9(1), 157169.

Denzin, N. K., \& Lincoln, Y. S. (2011). The Handbook of Qualitative Research. SAGE.

Dias Sobrinho, J. (2008). Calidad, pertinencia y responsabilidad social de la universidad latinoamericana y caribeña. Sao Paulo: A.L. Gazzola.

Duţă, N. V., \& Foloştină, R. (2014). Continuous Training of University Teachers - Theoretical Approaches and Practical Implications. Procedia - Social and Behavioral Sciences, 116, 3449-3453. https://doi.org/10.1016/j.sbspro.2014.01.781

Elton, L. (2006). Conservative Versus Innovative Universities. Personal Communication, (January 20.).

Fabela-Cárdenas, M. A., \& García-Treviño, A. H. (2014). Gestión de la calidad educativa en educación superior del sector privado. Quality Management in Private Higher Education., 6(13), 65-82. https://doi.org/10.11144/Javeriana.M6-13.GCEE

García Gallegos, K. H., Sarmiento Berrezueta, C. S. M., \& Rodríguez, M. J. (2016). Proceso de formación permanente del profesional de la educación superior en el contexto ecuatoriano. Process of Permanent Training of the Higher Education Professional in the Ecuadorian Context., 20(11), 5181-5184.

Gómez, V. G., Tolozano, M. R., \& Delgado, N. B. (2017). La Acreditación Institucional de la Calidad en los Institutos Superiores Técnicos y Tecnológicos del Ecuador desde la Perspectiva de un Instituto Acreditado. The Institutional Accreditation of Quality in Technical 
and Technological Institutes of Higher Education in Ecuador from the Perspective of an Accredited Institute., 10(6), 59-66. https://doi.org/10.4067/S0718-50062017000600007

González, H. G. (2014). Competences for Employability Among Trainee Teachers. Procedia Social and Behavioral Sciences, 139, 268-272. https://doi.org/10.1016/j.sbspro.2014.08.073

González, L. E., \& Espinoza, Ó. (2008). Calidad de la Educación Superior: Concepto y Modelos. Calidad En La Educación, (28), 248-276.

Granja, R. E. P. (2018). Procesos de evaluación de la educación superior y la implicación en las carreras de educación. Olimpia: Publicación científica de la facultad de cultura física de la Universidad de Granma, 15(49 (Abril-junio)), 276-289.

Hidalgo, L. G. A. (2011). La globalización de la educación superior y sus impactos en el Ecuador (http://purl.org/dc/dcmitype/Text). Universidad Pablo de Olavide. Retrieved from http://dialnet.unirioja.es/servlet/tesis?codigo=129696

Lara, F., \& Gascón, A. de la H. (2016). Reflexiones sobre la educación del sumak kawsay en Ecuador. Araucaria; $\quad$ Sevilla, 18(36), 41-58. http://dx.doi.org.puce.idm.oclc.org/10.12795/araucaria.2016.i36.03

Lemaître, M. J. (2005). Aseguramiento de calidad y reconocimiento en una perspectiva global. Estudios Sociales, (116), 9-24.

León, L. (2010). Sumak Kawsay/Buen Bivir y cambios civilizatorios. Quito: Fedaeps.

Mag, A. G. (2014). Challenges and Transformations in Teachers' Training. Procedia - Social and Behavioral Sciences, 128, 181-185. https://doi.org/10.1016/j.sbspro.2014.03.140

Marginson, S. (Ed.). (2016a). Higher Education and Society. In The Dream Is Over (pp. 178192). University of California Press. Retrieved from http://www.jstor.org/stable/10.1525/j.ctt1kc6k1p.26

Marginson, S. (Ed.). (2016b). The Uses of the University. In The Dream Is Over (pp. 21-27). University of California Press. Retrieved from http://www.jstor.org/stable/10.1525/j.ctt1kc6k1p.8

Martínez-Moscoso, A., \& Montesinos, P. V. (2012). La importancia de la evaluación en las instituciones educativas conforme a la Nueva Ley Orgánica de Educación Superior en el Ecuador. Revista Iberoamericana de Evaluación Educativa, 5(2), 174-180.

Mas, O., \& Tejada, J. (2013). Funciones y competencias en la docencia universitaria. Madrid: Síntesis.

Objetivos Nacionales para el Buen Vivir - Plan Nacional 2013 - 2017. (n.d.). Retrieved April 29, 2015, from http://www.buenvivir.gob.ec/objetivos-nacionales-para-el-buen-vivir

Patton, K., \& Parker, M. (2017). Teacher education communities of practice: More than a culture of collaboration. Teaching and Teacher Education, 67, 351-360. https://doi.org/10.1016/j.tate.2017.06.013

Ramírez, R. (2012). Transformar la universidad para transformar la sociedad. Quito: Secretaría

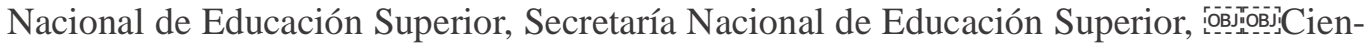
cia, Tecnología e Innovació.

Rodríguez, M. N., Aguilar, J., \& Apolo, D. (2018). El Buen Vivir como desafío en la formación de maestros: Aproximaciones desde la Universidad Nacional de Educación del Ecuador. Revista Mexicana de Investigación Educativa, 23(77), 577-596.

Rodríguez-Ponce, E., Pedraja-Rejas, L., Araneda-Guirriman, C., González-Plitt, M., \& Rodríguez-Ponce, J. (2011). El impacto del sistema de aseguramiento de la calidad en el servicio entregado por las universidades privadas en Chile/The impact of the quality assurance system in the service delivered by the private universities in Chile. Ingeniare: Revista Chilena de Ingenieria; Arica, 19(3), 409-419.

Rosal Durán, A. M. (2017). Reflexiones en torno a algunas ideas sobre la calidad de la enseñanza en la educación superior. Revista Cultura de Guatemala, 38(1), 101-109.

Ruiz, C., Mas, O., Tejada, J., \& Navío, A. (2008). Funciones y escenarios de actuación del profesor universitario. Apuntes para la definición del perfil basado en competencias. Revista de La Educación Superior, (146), 115-132.

Secretaria Nacional de Educación Superior, Ciencia y Tecnología. (2012). Transformar la Universidad para transformar la sociedad (Segunda). Quito. 
Semradova, I., \& Hubackova, S. (2014). Responsibilities and Competences of a University Teacher. Procedia - Social and Behavioral Sciences, 159, 437-441. https://doi.org/10.1016/j.sbspro.2014.12.403

SENPLADES. (2013). Plan Nacional del Buen Vivir 2013-2017. Quito.

Toscanini Segale, M., Aguilar Guzmán, A., \& García Sánchez, R. (2016). Diagnóstico de las políticas públicas de la educación superior en el Ecuador. Diagnosis of Higher Education’s Public Policies in Ecuador., 35(3), 161-178.

Vélez, C. M., \& Vélez León, P. (2008). Conceptos básicos de la evaluación externa a los programas de postgrado. IDEAS Working Paper Series from RePEc; St. Louis. Retrieved from http://search.proquest.com/docview/1698440792/668E96CBD75C49C7PQ/21

Villavicencio, A. (2012). Evaluación y acreditación en tiempos de cambio: La política pública universitaria en cuestionamiento. Quito: Instituto de Altos Estudios Nacionales.

Zlatić, L., Bjekić, D., Marinković, S., \& Bojović, M. (2014). Development of Teacher Communication Competence. Procedia - Social and Behavioral Sciences, 116, 606-610. https://doi.org/10.1016/j.sbspro.2014.01.265 